\title{
Quantum transport in a resonant tunnel junction coupled to a nanomechanical oscillator
}

\author{
M. Tahir* and A. MacKinnon \\ Department of Physics, Blackett Laboratory, Imperial College London, South Kensington Campus, London SW7 2AZ, United Kingdom
}

(Received 26 November 2007; revised manuscript received 2 June 2008; published 27 June 2008)

\begin{abstract}
We discuss the quantum transport of electrons through a resonant tunnel junction coupled to a nanomechanical oscillator at zero temperature. By using the Green's-function technique, we calculate the transport properties of electrons through a single dot strongly coupled to a single oscillator. We consider a finite chemicalpotential difference between the right and left leads. In addition to the main resonant peak of electrons on the dot, we find satellite peaks due to the creation of phonons. These satellite peaks become sharper and more significant with increasing coupling strength between the electrons and the oscillator. We also consider the energy transferred from the electrons to the oscillator.
\end{abstract}

DOI: 10.1103/PhysRevB.77.224305

PACS number(s): 73.23.Hk, 85.85.+j

\section{INTRODUCTION}

In recent years, there has been great interest in quantum transport through both single ${ }^{1}$ electronic levels in quantum dots and single molecules. ${ }^{2}$ Very interesting and significant signatures of the electron-phonon interaction have been observed experimentally ${ }^{3}$ in cases where the electron-phonon coupling is beyond the validity of simple perturbation theory. Single molecular electronics ${ }^{4}$ has also attracted much attention, motivated both by the scientific challenges and by their potential applications in nanoelectromechanical systems (NEMS)., 5 The most interesting challenges are the verification of the uncertainty principle, ${ }^{7}$ the study of the quantum transport properties as atomic scale quantum effects ${ }^{8,9}$ become more important and significant, and the fabrication of devices on the nanoscale that are expected to be faster, more reliable, and more sophisticated than existing technology. An interesting aspect of NEMS is the interplay between electrons, phonons, and the coupling of leads to the system. ${ }^{9,10}$ Inelastic tunneling spectroscopy ${ }^{11,12}$ is the most direct way of observing the inelastic effects in the current-voltage $(I-V)$ characteristics of NEMS. NEMS can be used as ultrasensitive detectors for mass, ${ }^{13}$ extremely weak forces, ${ }^{14}$ ultrasmall displacements, ${ }^{15}$ and even in chemical ${ }^{16}$ and biological ${ }^{17}$ applications. At very low bias, voltage resonances occur with the frequency of the nanomechanical oscillator. The characteristic frequency associated with these systems is on the order of $1 \mathrm{GHz} .{ }^{18}$ Such a high resonance frequency is sufficient to enable the cooling ${ }^{19}$ of a nanomechanical resonator to its ground state: a necessary condition for these measurements and something on which experimental effort is now under way. Moreover, the quantum transport requires very highly sensitive measurements that are achievable such as those using single electron transistors (SETs) or superconducting single electron transistors (SSETs).

Most of the theoretical work on transport in NEMS has been done within the scattering theory approach (Landauer), but it disregards the contacts and their effects on the scattering channel as well as effect of electrons and phonons on each other. ${ }^{20}$ Very recently, the nonequilibrium Green'sfunction (NEGF) approach ${ }^{21}$ has been growing in importance in the quantum transport of nanomechanical systems. ${ }^{22}$ An advantage of this method is that it treats the infinitely extended reservoirs in an exact way, which may lead to a better understanding of the essential features of NEMS. The pio- neering work on NEGF was done by Datta and Lake. ${ }^{23}$ NEGF has been applied in the study of shot noise in chain models ${ }^{24}$ and disordered junctions, ${ }^{25}$ while noise in Coulomb blockaded Josephson junctions has been discussed within a phase correlation theory approach. ${ }^{26}$ The case of an inelastic resonant tunneling structure, in which strong electronphonon coupling is often considered, a very strong sourcedrain voltage is expected for which coherent electron transport in molecular devices has been considered by some workers ${ }^{27}$ within the scattering theory approach. Inelastic effects on the transport properties have been studied in connection with NEMS and substantial work on this issue has been done, again within the scattering theory approach. ${ }^{20}$ Phononassisted tunneling of nonresonant systems has mostly been shown by experiments on inelastic tunneling spectroscopy (ITS). With the advancement of modern technology, as compared to ITS, scanning tunneling spectroscopy (STS) and scanning tunneling microscopy (STM) have proved to be more valuable tools for the investigation and characterization of molecular systems ${ }^{28}$ in the conduction regime. Recently, phonon-assisted resonant tunneling conductance has been discussed within the NEGF technique at zero temperature. ${ }^{29}$

In the present work, we employ the Green's-function method in order to discuss the transport properties of NEMS. This is a fully quantum-mechanical formulation whose basic approximations are very transparent, as the technique has already been used to study transport properties in a wide range of systems. The main differences between existing work and ours is that in most of the existing literature a very large chemical-potential difference is considered, while we consider ranges from the very small to the very large. In our calculation the inclusion of the oscillator is not perturbative as the STS experiments ${ }^{9,10}$ are beyond the range of perturbation theory. Hence, an approach is required beyond the quantum master equation ${ }^{22,30}$ or linear response. In addition, we aim in the future to apply the same methodology to describe more clearly nonperturbative systems such as a quantum shuttle. ${ }^{22}$ Hence, our work provides an exact analytical solution to the current-voltage characteristics, conductance, coupling of leads with the system, and very small chemicalpotential difference; and it includes both the right and left Fermi-level response regimes. However, our theory does rely on the commonly used wide-band approximation, ${ }^{31-33}$ where the coupling between leads and dot is taken to be independent of energy. This provides a way to perform transient 
transport calculations from first principles while retaining the essential physics of the electronic structure of the dot and the leads. Another advantage of this method is that it treats the infinitely extended reservoirs in an exact way in the present system, which may give a better understanding of the essential features of NEMS in a more appropriate quantummechanical picture.

\section{FORMULATION}

We consider a single quantum dot connected to two identical metallic leads. A single oscillator is coupled to the electrons on the dot and an applied gate voltage is used to tune the single level of the dot. In the present system, we neglect the spin degree of freedom and electron-electron interaction effects and consider the simplest possible model system. In this initial work, we also neglect the effects of finite electron temperature of the lead reservoirs and damping of the oscillator. Our model consists of the individual entities such as a single quantum dot and the left and right leads in their ground states at zero temperature. The Hamiltonian of our simple system ${ }^{29,31,32}$ is

$$
H_{\text {dot-ph }}=\left[\epsilon_{0}+\alpha\left(b^{\dagger}+b\right)\right] c_{0}^{\dagger} c_{0}+\omega_{0} b^{\dagger} b,
$$

where $\epsilon_{0}$ is the single energy level of electrons on the dot with $c_{0}^{\dagger}, c_{0}$ as the corresponding creation and annihilation operators, $\alpha$ is the coupling strength between electrons on the dot and an oscillator of frequency $\omega_{0}$, and $b^{\dagger}, b$ are the raising and lowering operators of the phonons. The remaining elements of the Hamiltonian are

$$
\begin{gathered}
H_{\text {leads }}=\sum_{k=L, R} \epsilon_{k} c_{k}^{\dagger} c_{k} \\
H_{\text {leads-dot }}=\frac{1}{\sqrt{N}} \sum_{k=L, R} V_{k}\left(c_{k}^{\dagger} c_{0}+c_{0}^{\dagger} c_{k}\right),
\end{gathered}
$$

where $N$ is the total number of states in each lead. The total Hamiltonian of the system is thus

$$
H=H_{\text {dot-ph }}+H_{\text {leads }}+H_{\text {leads-dot }} \cdot
$$

We write the eigenfunctions of $H_{\text {dot-ph }}$ in a $k$-space representation as

$$
\begin{gathered}
\Phi_{n}\left(k, x_{0} \neq 0\right)=A_{n} \exp \left(-\frac{1}{2} k^{2}\right) H_{n}(k) \exp \left(-\mathrm{i} k x_{0}\right), \\
\Phi_{n}\left(k, x_{0}=0\right)=A_{n} \exp \left(-\frac{1}{2} k^{2}\right) H_{n}(k)
\end{gathered}
$$

for the occupied, $x_{0} \neq 0$, and unoccupied, $x_{0}=0$, dots, respectively, where $x_{0}$ is the displacement of the oscillator due to the coupling to the electron and $H_{n}(k)$ are the usual Hermite polynomials. Here we have used the fact that the harmonic oscillator eigenfunctions have the same form in both real and Fourier space.

In order to transform between the representations for the occupied and unoccupied dots, we require the matrix with elements

$$
A_{m n}=\int \Phi_{n}^{*}\left(k, x_{0} \neq 0\right) \Phi_{n}\left(k, x_{0}=0\right) \mathrm{d} k,
$$

which may be simplified ${ }^{34}$ as

$$
\begin{aligned}
A_{m n} & =\frac{l}{\sqrt{\pi 2^{m+n} n ! m !}} \int \exp \left(-k^{2}\right) H_{m}(k) H_{n}(k) \exp \left(i k x_{0}\right) d k \\
& =\sqrt{\frac{2^{n-m} m !}{n !}} \exp \left(-\frac{1}{4} x_{0}^{2}\right)\left(\frac{1}{2} i x_{0}\right)^{|n-m|} L_{m}^{|n-m|}\left(\frac{1}{2} x_{0}^{2}\right)
\end{aligned}
$$

for $m \leq n$, where $x_{0}=\Delta / \omega_{0}, \Delta=\alpha^{2} / \omega_{0}$, and $L_{m}^{n}(x)$ are the associated Laguerre polynomials. Note that the integrand is symmetric in $m$ and $n$ but the integral is valid only for $m$ $\leq n$. Clearly the result for $m>n$ is obtained by exchanging $m$ and $n$ in Eq. (8) to obtain

$$
\begin{aligned}
A_{m n}= & \sqrt{\frac{2^{|n-m|} \min [m, n] !}{\max [m, n] !}} \\
& \times \exp \left(-\frac{1}{4} x_{0}^{2}\right)\left(\frac{1}{2} i x_{0}\right)^{|n-m|} L_{\min [m, n]}^{|n-m|}\left(\frac{1}{2} x_{0}^{2}\right) .
\end{aligned}
$$

\section{GREEN'S FUNCTIONS AND QUANTUM TRANSPORT}

In order to calculate analytical results and to discuss the numerical spectrum of the quantum transport properties of nanomechanical systems, our focus in this section is to derive an analytical relation for the current, as well as the differential conductance, as a function of applied bias. In obtaining these results, we use the wide-band approximation where the self-energy of the dot due to each lead is considered to be energy independent and is given by

$$
\Sigma_{K}^{r}=n_{D}\left|V_{K}\right|^{2} \int_{-\infty}^{+\infty} \frac{d \epsilon_{K}}{E-\epsilon_{K}}=-2 \pi i n_{D}\left|V_{K}\right|^{2}=-i \frac{1}{2} \Gamma_{K},
$$

where $n_{D}$ is the constant number density of the leads, $K$ $=L, R$ represent the left and right leads, and $\Gamma_{K}$ is the damping factor $\left(\Gamma_{L}=\Gamma_{R}=\Gamma\right)$. Similarly

$$
\Sigma_{K}^{a}=\left[\Sigma_{K}^{r}\right]^{*}=+i \frac{1}{2} \Gamma_{K}
$$

We solve Dyson's equation using $H_{\text {dot-leads }}$ as a perturbation. For the more general systems we aim to treat in the future, this is a reasonable small parameter. In the present case, however, we can find an exact solution. The retarded and advanced Green's functions on the dot, with the phonon states in the representation of the unoccupied dot, may be written as

$$
G_{n n^{\prime}}^{r(a)}=\sum_{m} A_{n m} g_{m}^{r(a)} A_{n^{\prime} m}^{*},
$$

where $g_{n}^{r(a)}$ is the retarded (advanced) Green's function on the occupied dot,

$$
g_{n}^{r(a)}=\left[E-\epsilon_{0}-\left(n+\frac{1}{2}\right) \omega_{0}+\Delta \pm i \Gamma\right]^{-1},
$$

with $\Delta=\alpha^{2} / \omega_{0}$.

The lesser self-energy may be written as

$$
\Sigma_{K}^{<}(E)=i \Gamma f_{K}(E)
$$

where $f_{L(R)}$ are the Fermi distribution functions of the left and right leads, which have different chemical potentials un- 
der a voltage bias, including a contribution from the state of the oscillator.

For the present case of zero temperature, the lesser selfenergy may be recast in terms of the Heaviside step function $\theta(x)$ as

$$
\Sigma_{K}^{<}(E)=i \Gamma \theta\left(\epsilon_{F K}+\frac{1}{2} \omega_{0}-E\right) \delta_{n, 0},
$$

where $\epsilon_{F K}$ is the Fermi energy on lead $K$ and the Kronecker delta, $\delta_{n, 0}$, signifies that the oscillator is initially in its ground state, $n=0$. Similarly one can calculate the greater selfenergy as

$$
\Sigma_{K}^{>}(E)=-i \Gamma\left[1-f_{K}(E)\right] .
$$

The lesser Green's function is related to the density matrix through

$$
\rho_{n n^{\prime}}=-2 i G_{n n^{\prime}}^{<} .
$$

Here $G_{n n^{\prime}}^{<}$is the full lesser Green's function including the dot and the leads. With the help of the density matrix formulation, the current formula is

$$
I=\langle\hat{I}\rangle=\operatorname{Tr}(\rho \hat{I})=i \frac{1}{2} \operatorname{Tr}\left(G^{<} \hat{I}\right),
$$

where $\hat{I}$ is the current operator. Using this formula, we calculate the current from the contact through both barriers and the central region with the oscillator on the dot. The general expression for the current is

$$
\begin{aligned}
I= & \frac{e}{4 \pi} \int\left\{\operatorname{Tr}\left[\left(\Sigma_{L}^{<}-\Sigma_{R}^{<}\right)\left(G^{r}-G^{a}\right)\right]\right. \\
& \left.+\operatorname{Tr}\left[\left(\left(\Sigma_{L}^{a}-\Sigma_{L}^{r}\right)-\left(\Sigma_{R}^{a}-\Sigma_{R}^{r}\right)\right) G^{<}\right]\right\} \mathrm{d} E .
\end{aligned}
$$

We can obtain the same result by calculating the current from the dot into one of the leads, which gives

$$
I=\frac{e}{4 \pi} \int\left\{\operatorname{Tr}\left[-\Sigma_{R}^{<}\left(G^{r}-G^{a}\right)\right]-\operatorname{Tr}\left[\left(\Sigma_{R}^{a}-\Sigma_{R}^{r}\right) G^{<}\right]\right\} \mathrm{d} E,
$$

where the first term in the above expression vanishes for $n$ $>0$. The lesser Green's function in the presence of the oscillator is given by

$$
G^{<}=G^{r} \Sigma^{<} G^{a} \quad \text { with } \quad \Sigma^{<}=\Sigma_{L}^{<}+\Sigma_{R}^{<} .
$$

Using the same damping factor in each lead $\left(\Gamma_{L}=\Gamma_{R}\right.$ $=\Gamma)$, the final expression for the total current $(I)$ reduces to

$$
I=\frac{e}{4 \pi} \int \operatorname{Tr}\left(\Sigma_{L}^{<}-\Sigma_{R}^{<}\right)\left(G^{r}-G^{a}\right) \mathrm{d} E
$$

\section{AVERAGE ENERGY}

To calculate the energy transferred from the electrons to the oscillator, we return to Eq. (20) and note that the contributions to the trace with $n>0$ may be identified with the current due to those electrons which have been scattered inelastically with the creation of $n$ phonons. As the lesser selfenergy factors in the first term are zero for $n>0$, the inelastic contributions are solely contained in the second term. The first term in Eq. (20) does contribute to the total current calculated in Eq. (22) but does not contribute to the energy of the oscillator. We may therefore use the lesser Green's function (or density matrix) to calculate the energy transferred to the oscillator to obtain

$$
E_{\mathrm{ph}}=\sum_{n} \frac{n \omega_{0} Y_{n}}{I} \quad \text { where } Y_{n}=\frac{1}{2} i \frac{\Gamma e}{4 \pi} \int G_{n n}^{<} d E .
$$

From Eq. (23) we may write the lesser Green's function in terms of the lesser self-energy and the retarded and advanced Green's functions as

$$
G_{n n}^{<}=G_{n 0}^{r}\left(\Sigma_{0, L}^{<}+\Sigma_{0, R}^{<}\right) G_{0 n}^{a},
$$

where we note that as we work at $T=0$, the self-energy terms are nonzero only for the zero-phonon state. Hence we have

$$
\begin{aligned}
Y_{n}= & \frac{1}{2} i \frac{\Gamma e}{4 \pi} \sum_{m, k} \int_{-\infty}^{\infty}\left[\frac{A_{n m} A_{0 m}^{*}}{E-\epsilon_{0}-\left(m+\frac{1}{2}\right) \omega_{0}+\Delta+i \Gamma}\right] \\
& \times\left[i \Gamma \theta\left(\epsilon_{F L}+\frac{1}{2} \omega_{0}-E\right)+i \Gamma \theta\left(\epsilon_{F R}+\frac{1}{2} \omega_{0}-E\right)\right] \\
& \times\left[\frac{A_{0 k} A_{n k}^{*}}{E-\epsilon_{0}-\left(k+\frac{1}{2}\right) \omega_{0}+\Delta-i \Gamma}\right] d E .
\end{aligned}
$$

We note, however, that this expression is nonzero even when $\epsilon_{F L}=\epsilon_{F R}$ and $\epsilon_{0}<\epsilon_{F}$ due to the dot being permanently occupied in these circumstances. To remove this term, we subtract the contribution when the two Fermi levels are equal. This reduces the expression for $Y_{n}$ to

$$
Y_{n}=-\frac{1}{2} \frac{\Gamma^{2} e}{4 \pi} \int_{\epsilon_{F R}}^{\epsilon_{F L}}\left|\sum_{m} \frac{A_{n m} A_{0 m}^{*}}{E-\epsilon_{0}-\left(m+\frac{1}{2}\right) \omega_{0}+\Delta+i \Gamma}\right|^{2} d E .
$$

After integrating the above expression, ${ }^{35}$ we arrive at the final result,

$$
\begin{aligned}
Y_{n}= & -\frac{1}{2} \frac{\Gamma^{2} e}{4 \pi} \sum_{m, k} \frac{A_{n, m} A_{0, m}^{*} A_{0, k} A_{n, k}^{*}}{(k-m) \omega_{0}+2 i \Gamma} \\
& \times\left\{\ln \left[\frac{\epsilon_{F L}-\epsilon_{0}-m \omega_{0}+\Delta-i \Gamma}{\epsilon_{F L}-\epsilon_{0}-k \omega_{0}+\Delta+i \Gamma}\right]\right. \\
& \left.-\ln \left[\frac{\epsilon_{F R}-\epsilon_{0}-m \omega_{0}+\Delta-i \Gamma}{\epsilon_{F R}-\epsilon_{0}-k \omega_{0}+\Delta+i \Gamma}\right]\right\} .
\end{aligned}
$$

Hence, the average energy transferred to the oscillator may be calculated using Eq. (23).

\section{DISCUSSION OF RESULTS}

The differential conductance is shown graphically in Fig. 1 as a function of applied voltage for different values of coupling strength, using the following parameters: ${ }^{29,31,32,36-38}$ the single energy level of the dot $\epsilon_{0}=0.5$, the characteristic frequency of the oscillator $\omega_{0}=0.3$, the damping factor $\Gamma$ $=0.3 \omega_{0}$, and the chemical potentials $0 \leq \epsilon_{F L} \leq 1$ and $\epsilon_{F R}=0$. These are chosen to illustrate the physics of such systems 


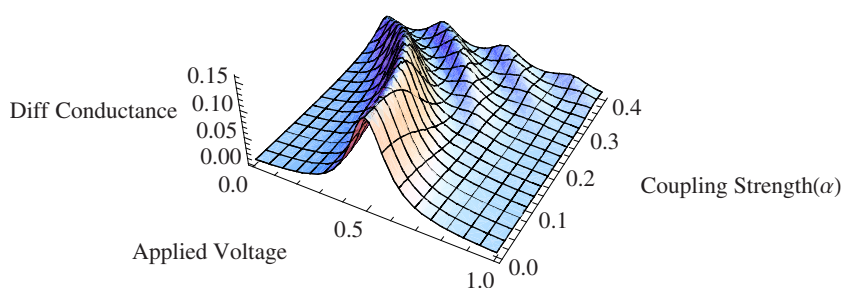

FIG. 1. (Color online) Differential conductance (dimensionless) as a function of applied voltage $\epsilon_{F L}$ (in arbitrary units) and coupling strength $\alpha$. Gate voltage $\epsilon_{0}=0.5$, oscillator frequency $\omega_{0}=0.3$, selfenergy $\Gamma=0.3 \omega_{0}$.

rather than to represent a specific implementation. The oscillator induced resonance effects are clearly visible in the numerical results. It must be noted that we have obtained these results in the regime of strong and zero or weak coupling of the oscillator with the electrons on the dot. The coupling between the leads and the dot is considered to be symmetric and we assume that the leads have constant density of states. With increasing coupling strength, the number of satellite peaks also increases, while for zero or weak coupling we find only the basic resonance. This confirms the effect of the coupling between the electrons on the dot and the single oscillator mode where higher-energy electrons are able to drop to the dot energy by creation of phonons. We note the similarity of Figs. 1-3(a) of Ref. 39, which refers, however, to the transmission amplitude of an interference device, albeit using a similar Hamiltonian. Transport processes involving creation or annihilation of phonons are a common feature of NEMS.

Closer analytical examination of the expression for differential conductance (26) shows that the main resonance peaks occur when the applied voltage $\epsilon_{F L}$ is equal to the energy eigenvalues of the coupled dot electron and oscillator. The main peak $(n=0)$ is given by the Lorentzian form with its center at $\epsilon_{F L}=\epsilon_{0}-\Delta$, known as a Breit-Wigner ${ }^{36,40,41}$ resonance. The satellite peaks due to the emission of phonons can be seen on the positive energy side with $\epsilon_{F L}=\epsilon_{0}-\Delta$ $+n \omega_{0}$, where $\omega_{0}$ is the characteristic frequency of the oscillator.

The main or basic resonance peak is the elastic or zerophonon transition. The amplitude of the satellite peaks or steps is much smaller than the basic resonance peak. The electrons that tunnel into the dot can excite only the oscillator mode as at zero temperature there are no phonons available to be absorbed. Moreover, we have seen that with increasing coupling strength, the number and intensity of the satellite peaks increases but their intensity always remains much smaller than the main peak. The peaks or steps in the current characteristics vanish if the upper electrochemical potential is smaller than the dot energy plus the oscillator frequency.

The differential conductance as a function of gate voltage $\epsilon_{0}$ is shown in Fig. 2 for various coupling strengths at $T=0$. The main peak at $\epsilon_{0}=\epsilon_{F L}+\Delta$ corresponds to elastic or zerophonon transition and the satellites peaks are due to emission of phonons corresponding to $n=1,2,3,4, \ldots$. This shows more and more satellites corresponding to every multiple of $\omega_{0}$.

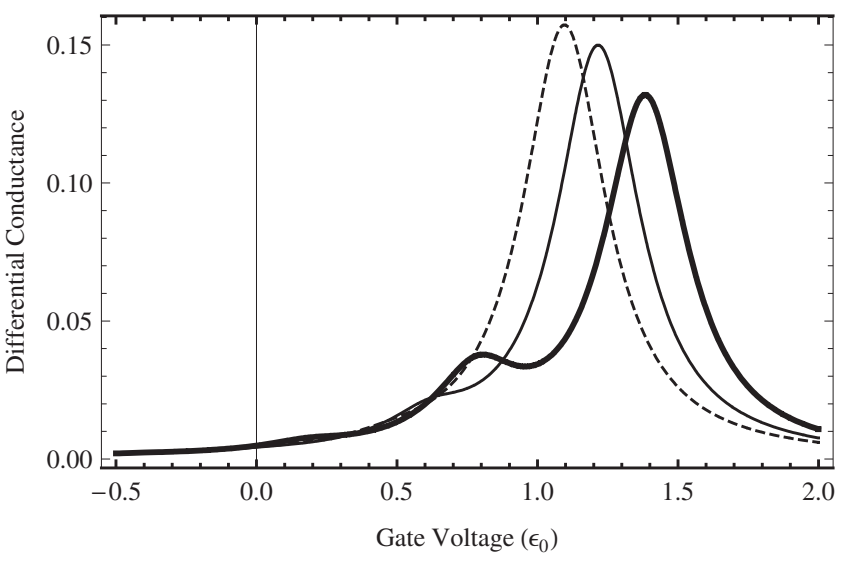

FIG. 2. Differential conductance (dimensionless) as a function of gate voltage $\epsilon_{0}$, with applied voltage $\epsilon_{F L}=1$, oscillator frequency $\omega_{0}=0.6$, self-energy $\Gamma=0.3 \omega_{0}$, and coupling strength $\alpha=0.4 \omega_{0}$ (dotted line), $0.6 \omega_{0}$ (light solid line), and $0.8 \omega_{0}$ (bold solid line).

With increasing coupling strength at constant zero temperature, we see that the energy transferred to the oscillator increases, while the amplitude of the satellite peaks is much smaller than the main peak, which is shifted toward the right by a factor of $\Delta$. The amplitude of the main peak is also affected: its magnitude decreases with increasing coupling strength.

In Fig. 3, we plot the average energy that is transferred to the oscillator per transmitted electron as a function of applied bias and gate voltage. At zero temperature the oscillator can only gain energy from the electrons. We note that there is some structure as individual phonons are excited but there is also a saturation level $\propto \alpha^{4}$. The peak just below $\epsilon_{0} \approx \epsilon_{F R}$ in the figure at right is due to the fact that there is no elastic transmission in this regime and all transmitted electrons result in the creation of phonons. Note that this is the average energy transferred when the system starts in its ground state and should not be confused with the energy transferred after many electrons have interacted with the oscillator. We consider $T=0$ in this work for simplicity and will consider finite temperature effects later. Moreover, the phonon energy of the oscillator on the dot and the level width are both typically larger than the experimental temperature. Our theory is in good agreement with the growing body of theoretical $29,31,32,36$ and experimental ${ }^{37,38}$ works in this area.

\section{SUMMARY}

In this work, we analyzed the dynamics of a nanomechanical oscillator coupled to a resonant tunnel junction by using the Green's-function approach without treating the electron-phonon coupling as a perturbation. We have derived an expression for the current and differential conductance and discuss it in detail for different values of the coupling strength. We have found steps/peaks in the current spectrum as a function of the chemical-potential difference in addition to the main resonant step, due to the transfer of energy from electrons on the dot to the oscillator. We have also studied the effect of gate voltage. We have also derived an expres- 

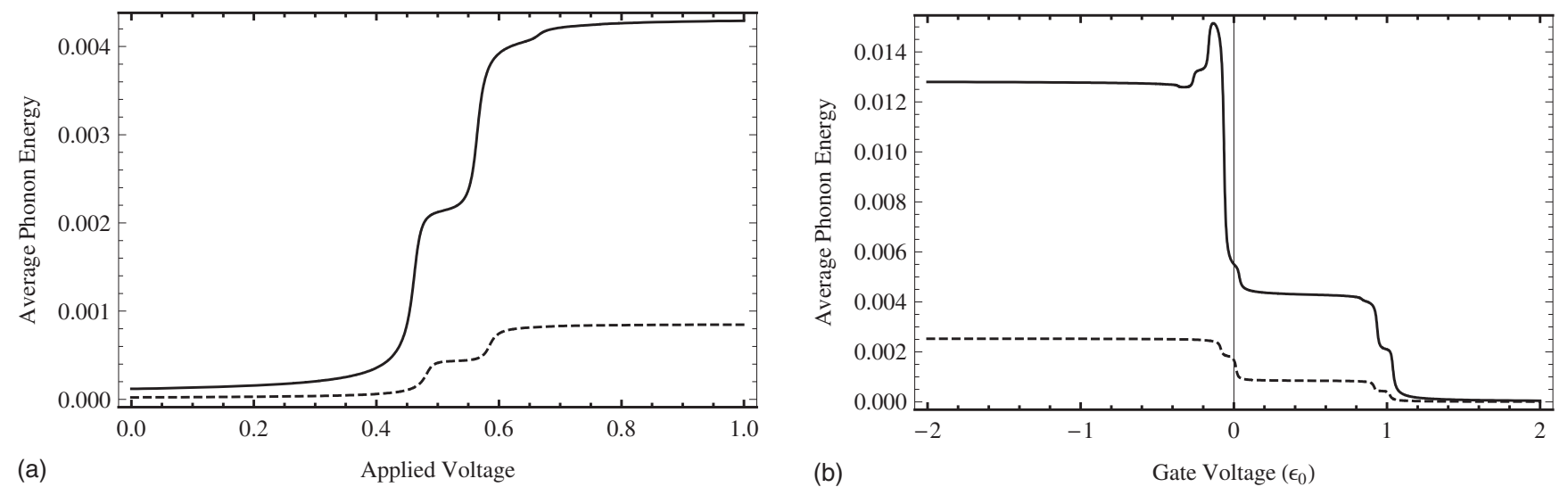

FIG. 3. Average energy transferred to the oscillator as a function of applied voltage $\epsilon_{F L}$ with gate voltage $\epsilon_{0}=0.5$ (left figure) and as a function of gate voltage $\epsilon_{0}$ with $\epsilon_{F L}=1$ (right figure). Oscillator frequency $\omega_{0}=0.1$, self-energy $\Gamma=0.1 \omega_{0}$, and coupling strength $\alpha=0.4 \omega_{0}$ (dotted line), and $\alpha=0.6 \omega_{0}$ (solid line).

sion for the average energy transferred from the electrons to the oscillator. We have shown that the steps grow with increasing coupling strength of electrons on the dot and the oscillator. This confirms that the additional satellite peaks or steps in the spectrum of numerical results are due to the transfer of energy from the electrons to the oscillator.

\section{ACKNOWLEDGMENT}

M.T. would like to acknowledge the support of the Pakistan Higher Education Commission (HEC).
* Permanent address: Department of Physics, University of Sargodha, Sargodha 40100, Pakistan; m.tahir06@imperial.ac.uk, m.tahir@uos.edu.pk

${ }^{1}$ J. Koch and F. von Oppen, Phys. Rev. Lett. 94, 206804 (2005); J. Koch, M. E. Raikh, and F. von Oppen, ibid. 95, 056801 (2005); J. Koch, F. von Oppen, and A. V. Andreev, Phys. Rev. B 74, 205438 (2006).

${ }^{2}$ M. A. Reed, C. Zhou, C. J. Muller, T. P. Burgin, and J. M. Tour, Science 278, 252 (1997); H. Park, J. Park, A. K. L. Lim, E. H. Anderson, A. P. Alivisatos, and P. L. McEuen, Nature (London) 407, 57 (2000); R. H. M. Smit, Y. Noat, C. Untiedt, N. D. Lang, M. C. van Hemert, and J. M. van Ruitenbeek, ibid. 419, 906 (2002); L. H. Yu, Z. K. Keane, J. W. Ciszek, L. Cheng, M. P. Stewart, J. M. Tour, and D. Natelson, Phys. Rev. Lett. 93, 266802 (2004); L. H. Yu and D. Natelson, Nano Lett. 4, 79 (2004); M. Elbing, R. Ochs, M. Koentopp, M. Fischer, C. von Hänisch, F. Weigend, F. Evers, H. B. Weber, and M. Mayor, Proc. Natl. Acad. Sci. U.S.A. 102, 8815 (2005); M. Poot, E. Osorio, K. O’Neill, J. M. Thijssen, D. Vanmaekelbergh, C. A. van Walree, L. W. Jenneskens, and H. S. J. van der Zant, Nano Lett. 6, 1031 (2006); E. A. Osorio, K. O’Neill, N. Stuhr-Hansen, O. F. Nielsen, T. Bjèrnholm, and H. S. J. van der Zant, Adv. Mater. (Weinheim, Ger.) 19, 281 (2007); E. Lörtscher, H. B. Weber, and H. Riel, Phys. Rev. Lett. 98, 176807 (2007).

${ }^{3}$ X. H. Qiu, G. V. Nazin, and W. Ho, Phys. Rev. Lett. 92, 206102 (2004); J. Repp, G. Meyer, S. M. Stojković, A. Gourdon, and C. Joachim, ibid. 94, 026803 (2005); J. Repp, G. Meyer, S. Paavilainen, F. E. Olsson, and M. Persson, ibid. 95, 225503 (2005).

${ }^{4}$ G. Cuniberti, G. Fagas, and K. Richter, Introducing Molecular Electronics (Springer-Verlag, Berlin, 2005); M. A. Reed and J. M. Tour, Sci. Am. 282 (6), 86 (2000); C. Joachim, J. K. Gimze- wski, and A. Aviram, Nature (London) 408, 541 (2000).

${ }^{5}$ K. L. Ekinci and M. L. Roukes, Rev. Sci. Instrum. 76, 061101 (2005); K. L. Ekinci, Small 1, 786 (2005); M. L. Roukes, Technical Digest of the 2000 Solid State Sensor and Actuator Workshop (unpublished); H. G. Craighead, Science 290, 1532 (2000); P. Kim and C. M. Lieber, ibid. 286, 2148 (1999); S. Akita, Y. Nakayama, S. Mizooka, Y. Takano, T. Okawa, Y. Miyatake, S. Yamanaka, M. Tsuji, and T. Nosaka, Appl. Phys. Lett. 79, 1691 (2001); A. M. Fennimore, T. D. Yuzvlnsky, W. Q. Han, M. S. Fuhrer, J. Cummings, and A. Zettl, Nature (London) 424, 408 (2003).

${ }^{6}$ J. Kinaret, T. Nord, and S. Viefers, Appl. Phys. Lett. 82, 1287 (2003); C.-H. Ke and H. D. Espinosa, ibid. 85, 681 (2004); V. Sazonova, Y. Yaish, H. Üstünel, D. Roundy, T. Arias, and P. McEuen, Nature (London) 431, 284 (2004).

${ }^{7}$ Miles Blencowe, Nature (London) 424, 262 (2003); Ivar Martin, Alexander Shnirman, Lin Tian, and Peter Zoller, Phys. Rev. B 69, 125339 (2004).

${ }^{8}$ Miles Blencowe, Science 304, 56 (2004).

${ }^{9}$ M. D. LaHaye, O. Buu, B. Camarota, and K. C. Schwab, Science 304, 74 (2004); K. C. Schwab and M. L. Roukes, Phys. Today 58 (7), 36 (2005).

${ }^{10}$ S. W. Wu, G. V. Nazin, X. Chen, X. H. Qiu, and W. Ho, Phys. Rev. Lett. 93, 236802 (2004).

${ }^{11}$ B. C. Stipe, M. A. Rezaei, and W. Ho, Rev. Sci. Instrum. 70, 137 (1999); Phys. Rev. Lett. 82, 1724 (1999); J. R. Hahn, H. J. Lee, and W. Ho, ibid. 85, 1914 (2000).

${ }^{12}$ W. Wang, T. Lee, and M. A. Reed, Rep. Prog. Phys. 68, 523 (2005); J. Phys. Chem. B 108, 18398 (2004); W. Wang, T. Lee, I. Kretschmar, and M. A. Reed, Nano Lett. 4, 643 (2004); J. G. Kushmerick, D. L. Allara, T. E. Mallouk, and T. S. Mayer, MRS Bull. 29, 396 (2004); J. G. Kushmerick, J. Lazorcik, C. H. 
Patterson, R. Shashidhar, D. S. Seferos, and G. C. Bazan, Nano Lett. 4, 639 (2004); Y. Selzer, M. A. Cabassi, T. S. Mayer, and D. L. Allara, Nanotechnology 15, S483 (2004).

${ }^{13}$ K. L. Ekinci, Appl. Phys. Lett. 84, 4469 (2004); Y. T. Yang, ibid. 78, 162 (2001); X. M. H. Huang, C. A. Zorman, M. Mehregany, and M. L. Roukes, Nature (London) 421, 496 (2003); B. Ilic, D. Czaplewski, H. G. Craighead, P. Neuzil, C. Campagnolo, and C. Batt, Appl. Phys. Lett. 77, 450 (2000); B. J. Costello, S. W. Wenzel, and R. M. White, Science 251, 1372 (1991); Y. T. Yang, Nano Lett. 6, 583 (2006); B. Ilic, H. G. Craighead, S. Krylov, W. Senaratne, C. Ober, and P. Neuzil, J. Appl. Phys. 95, 3694 (2004); K. L. Ekinci, Y. T. Yang, and M. L. Roukes, ibid. 95, 2682 (2004).

${ }^{14}$ H. J. Mamin and D. Rugar, Appl. Phys. Lett. 79, 3358 (2001).

${ }^{15}$ C. M. Caves, K. S. Thorne, R. W. P. Drever, V. D. Sandberg, and M. Zimmermann, Rev. Mod. Phys. 52, 341 (1980); V. B. Braginsky and F. Ya. Khalili Quantum Measurement (Cambridge University Press, Cambridge, 1992); M. F. Bocko and R. Onofrio, Rev. Mod. Phys. 68, 755 (1996); M. P. Blencowe and M. N. Wybourne, Appl. Phys. Lett. 77, 3845 (2000); R. G. Knobel and A. N. Cleland, Nature (London) 424, 291 (2003); R. G. Beck, M. A. Eriksson, M. A. Topinka, R. M. Westervelt, K. D. Maranowski, and A. C. Gossard, Appl. Phys. Lett. 73, 1149 (1998); R. Knobel and A. N. Cleland, ibid. 81, 2258 (2002); Y. Zhang and M. P. Blencowe, J. Appl. Phys. 92, 7550 (2002).

${ }^{16}$ E. A. Wachter and T. Thundat, Rev. Sci. Instrum. 66, 3662 (1995); H. P. Lang, R. Berger, C. Andreoli, J. Brugger, M. Despont, P. Vettiger, Ch. Gerber, J. K. Gimzewski, J. P. Ramseyer, E. Meyer, and H.-J. Guntherodt, Appl. Phys. Lett. 72, 383 (1998); Z. Hu, T. Thundat, and R. J. Warmack, J. Appl. Phys. 90, 427 (2001).

${ }^{17}$ B. Ilic, D. Czaplewski, H. G. Craighead, P. Neuzil, C. Campagnolo, and C. Batt, Appl. Phys. Lett. 77, 450 (2000); J. Fritz, M. K. Baller, H. P. Lang, H. Rothuizen, P. Vettiger, E. Meyer, H.-J. Guntherodt, Ch. Gerber, and J. K. Gimzewski, Science 288, 316 (2000); B. Ilic, D. Czaplewski, M. Zalalutdinov, H. G. Craighead, P. Neuzil, C. Campagnolo, and C. Batt, J. Vac. Sci. Technol. B 19, 2825 (2001); A. Subramanian, P. I. Oden, S. J. Kennel, K. B. Jacobson, R. J. Warmack, T. Thundat, and M. J. Doktycz, Appl. Phys. Lett. 81, 385 (2002); L. A. Pinnaduwage, V. Boiadjiev, J. E. Hawk, and T. Thundat, ibid. 83, 1471 (2003).

${ }^{18}$ J. Chen, M. Reed, A. Rawlett, and J. Tour, Science 286, 1550 (1999); M. Keil and H. Schoeller, Phys. Rev. B 66, 155314 (2002).

${ }^{19}$ A. Naik, O. Buu, M. D. LaHaye, A. D. Armour, A. A. Clerk, M. P. Blencowe, and K. C. Schwab, Nature (London) 443, 193 (2006).

${ }^{20}$ A. Shimizu and M. Ueda, Phys. Rev. Lett. 69, 1403 (1992); O. L. Bo and Yu. Galperin, Phys. Rev. B 55, 1696 (1997); B. Dong, H. L. Cui, X. L. Lei, and N. J. M. Horing, ibid. 71, 045331 (2005); Y.-C. Chen and M. Di Ventra, Phys. Rev. Lett. 95, 166802 (2005).

${ }^{21}$ L. V. Keldysh, Zh. Eksp. Teor. Fiz. 47, 1515 (1965); H. Huag and A. P. Jauho, Quantum Kinetics in Transport and Optics of Semiconductors, Springer Solid-State Sciences Vol. 123 (Springer, New York, 1996).

${ }^{22}$ N. Nishiguchi, Phys. Rev. Lett. 89, 066802 (2002); A. Yu. Smirnov, L. G. Mourokh, and N. J. M. Horing, Phys. Rev. B 67, 115312 (2003); A. A. Clerk and S. M. Girvin, ibid. 70, 121303(R) (2004); T. Novotný, A. Donarini, C. Flindt, and A.-P.
Jauho, Phys. Rev. Lett. 92, 248302 (2004); A. D. Armour and A. MacKinnon, Phys. Rev. B 66, 035333 (2002); C. Flindt, T. Novotny, and A.-P. Jauho, ibid. 70, 205334 (2004); J. Wabnig, D. V. Khomitsky, J. Rammer, and A. L. Shelankov, ibid. 72, 165347 (2005).

${ }^{23}$ S. Datta, J. Phys.: Condens. Matter 2, 8023 (1990); R. Lake and S. Datta, Phys. Rev. B 45, 6670 (1992); 46, 4757 (1992).

${ }^{24}$ J. Aghassi, A. Thielmann, M. H. Hettler, and G. Schön, Appl. Phys. Lett. 89, 052101 (2006); M. Kindermann and P. W. Brouwer, Phys. Rev. B 74, 125309 (2006).

${ }^{25}$ D. B. Gutman and Y. Gefen, Phys. Rev. B 64, 205317 (2001).

${ }^{26}$ E. B. Sonin, Phys. Rev. B 70, 140506(R) (2004); E. B. Sonin, J. Low Temp. Phys. 146, 161 (2007).

${ }^{27}$ S. Dallakyan and S. Mazumdar, Appl. Phys. Lett. 82, 2488 (2003); K. Walczak, Phys. Status Solidi B 241, 2555 (2004); Y.-C. Chen and M. Di Ventra, Phys. Rev. B 67, 153304 (2003); J. Lagerqvist, Y.-C. Chen, and M. Di Ventra, Nanotechnology 15, S459 (2004).

${ }^{28}$ T. Seideman, J. Phys.: Condens. Matter 15, R521 (2003); N. Lorente, R. Rurali, and H. Tang, ibid. 17, S1049 (2005); A. B. Migdal, Sov. Phys. JETP 7, 996 (1958); G. M. Eliashberg, ibid. 11, 696 (1960); A. L. Fetter and J. D. Walecka, Quantum Theory of Many-Particle Systems (Dover, New York, 2003).

${ }^{29}$ V. Aji, J. E. Moore, and C. M. Varma, arXiv:cond-mat/0302222 (unpublished); D. A. Ryndyk and G. Cuniberti, Phys. Rev. B 76, 155430 (2007); J. X. Zhu and A. V. Balatsky, ibid. 67, 165326 (2003).

${ }^{30}$ G. Kiesslich, E. Schöll, T. Brandes, F. Hohls, and R. J. Haug, Phys. Rev. Lett. 99, 206602 (2007); H. Hübener and T. Brandes, ibid. 99, 247206 (2007).

${ }^{31}$ Michael Galperin, Abraham Nitzan, and Mark A. Ratner, Phys. Rev. B 74, 075326 (2006); 73, 045314 (2006); V. Nam Do, P. Dollfus, and V. Lien Nguyen, Appl. Phys. Lett. 91, 022104 (2007).

${ }^{32}$ M. Galperin, M. A. Ratner, and A. Nitzan, J. Chem. Phys. 121, 11965 (2004); Nano Lett. 4, 1605 (2004).

${ }^{33}$ Ned S. Wingreen, Karsten W. Jacobsen, and John W. Wilkins, Phys. Rev. B 40, 11834 (1989).

${ }^{34}$ I. S. Gradshteyn and I. M. Ryzhik, Tables of Integrals, Series and Products (Academic, New York, 1980), p. 837.

${ }^{35}$ I. S. Gradshteyn and I. M. Ryzhik, Tables of Integrals, Series and Products (Academic, New York, 1980), pp. 66-67.

${ }^{36}$ J. Paaske and K. Flensberg, Phys. Rev. Lett. 94, 176801 (2005); K. Flensberg, Phys. Rev. B 68, 205323 (2003); Urban Lundin and Ross H. McKenzie, ibid. 66, 075303 (2002); A. Yacoby, M. Heiblum, D. Mahalu, and H. Shtrikman, Phys. Rev. Lett. 74, 4047 (1995).

${ }^{37}$ J. Park, A. Pasupathy, J. Goldsmith, C. Chang, Y. Yaish, J. Petta, M. Rinkoski, J. Sethna, H. Abruna, and P. McEuen, Nature (London) 417, 722 (2002).

${ }^{38}$ W. Liang, M. Shores, M. Bockrath, J. Long, and H. Park, Nature (London) 417, 725 (2002).

${ }^{39}$ A. D. Armour and M. P. Blencowe, Phys. Rev. B 64, 035311 (2001).

${ }^{40}$ L. D. Landau and E. M. Lifshiftz, Quantum Mechanics Nonrelativistic Theory (Pergamon, New York, 1977), p. 613.

${ }^{41}$ R. Schuster, E. Buks, M. Heiblum, D. Mahalu, V. Umansky, and H. Shtrikman, Nature (London) 385, 417 (1997); Akiko Ueda and Miko Eto, Phys. Rev. B 73, 235353 (2006). 